\title{
ANALYTICAL 3D BOUNDARY ELEMENT IMPLEMENTATION OF FLAT TRIANGLE AND QUADRILATERAL ELEMENTS FOR POTENTIAL AND LINEAR ELASTICITY PROBLEMS
}

\author{
NEY AUGUSTO DUMONT \& TATIANA GALVÃO KURZ \\ Department of Civil and Environmental Engineering, \\ Pontifical Catholic University of Rio de Janeiro, Brazil
}

\begin{abstract}
This paper introduces a formulation for 3D potential and linear elasticity problems that end up with the analytical handling of all regular, improper, quasi-singular, singular and hypersingular integrals of an implementation using linear triangle (T3) elements. The extension to flat Q4 and T6 elements is almost straightforward. Results at arbitrarily located internal points are also given analytically. The formulation is based on a generalized transformation to subtriangle coordinates that simplifies the problem's description and enables the adequate interpretation of all relevant geometric features of a discretized boundary segment, so that it becomes possible to arrive at manageable analytical expressions of all integrals. The paper outlines the main concepts and computational features of the proposed formulation, based on an array with all pre-evaluated integrals required in an implementation. An example of 3D potential problems illustrates all particular cases and the most challenging topological configurations one might deal with in practical applications. The procedure may be easily implemented in a general boundary element code, as the usual numerical quadrature schemes for source points sufficiently far from the integration field remain applicable. There is a work in progress for the implementation of the procedure in the frame of a fast multipole algorithm.

Keywords: collocation boundary element method, numerical integration, analytical integration, 3 D problems.
\end{abstract}

\section{INTRODUCTION}

The collocation boundary element method (BEM) [1] has been recently reconceptualized by the first author for general 2D and 3D problems [2], with the proposition of a general, simple and unified procedure for the machine-precision treatment of all kinds of singularities that may occur in 2D steady-state potential and elastostatics problems, as an extension of [3], [4].

The numeric issues for 3D problems are of a completely different nature [5]. The triangle-to-square coordinate transformation for the evaluation of improper integrals seems to have been firstly suggested for BEM applications in [6].

Simple analytical results are arrived at for all kinds of integrals required for 3D steadystate potential and elastostatics problems - for the boundary discretized with flat segments. This includes the evaluation of results at arbitrarily placed internal points, with hypersingularities also dealt with analytically. The case of a generally curved boundary segment is still not considered by the authors - and may not be mastered in the present framework. Although the proposed developments apply directly to elastostatics problems, space restrictions force us to draw attention only to potential problems.

\section{PROBLEM FORMULATION}

The following developments, as outlined in [5], apply to the adequate evaluation of integrals that embed improper, singular or quasi-singularities. The source point 0 is 
indicated in Fig. 1(c), where $r=\sqrt{\left(x-x_{0}\right)^{2}+\left(y-y_{0}\right)^{2}+\left(z-z_{0}\right)^{2}}$ is the distance from the singularity ( 0 or source) point $\left(x_{0}, y_{0}, z_{0}\right) \equiv\left(x\left(\xi_{s}, \eta_{s}\right), y\left(\xi_{s}, \eta_{s}\right), z\left(\xi_{s}, \eta_{s}\right)\right)$ to a generic (F or field) point $(x, y, z) \equiv(x(\xi, \eta), y(\xi, \eta), z(\xi, \eta))$ in Cartesian coordinates, expressed in terms of the indicated natural triangle coordinates $(\xi \in[0,1-\eta], \eta \in[0,1])$ in Fig. 1(a) as well as $(\xi, \eta \in[0,1])$ for the quadrilateral in the centre.

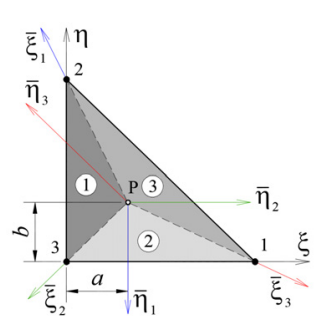

(a)

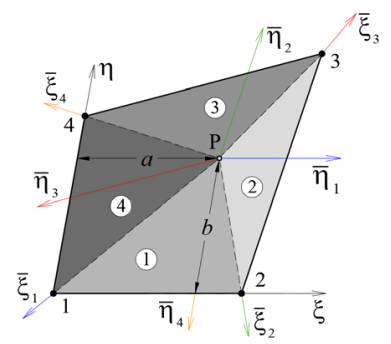

(b)

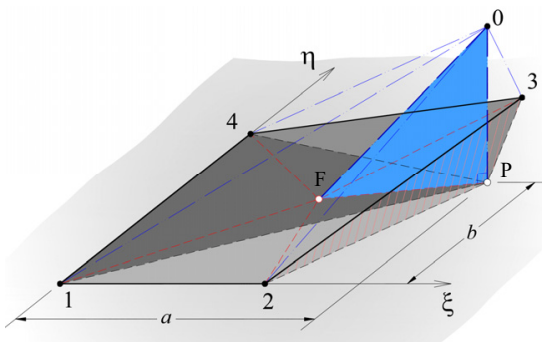

(c)

Figure 1: (a) Triangle; and (b) Quadrilateral elements described with two different systems of natural coordinates, $(\xi, \eta)$ and $\left(\bar{\xi}_{l}, \bar{\eta}_{l}\right)$, for an in-plane singularity $\mathrm{P}(\xi=a, \eta=b)$; (c) Shows the general problem in the Cartesian space for a quadrilateral element with a quasi-singular source 0 .

According to this proposition, the coordinates $(\xi, \eta)$ are linear transformations of $\left(\bar{\xi}_{l}, \bar{\eta}_{l}\right)$, as for a triangle element [5],

$$
\begin{gathered}
{[\xi, \eta]_{1}=\left[a\left(1-\bar{\xi}_{1}\right), \bar{\xi}_{1}\left(1-b-\bar{\eta}_{1}\right)+b\right] ; \quad|J|_{1}=\bar{\xi}_{1} a \text { for } \Delta_{1},} \\
{[\xi, \eta]_{2}=\left[\bar{\xi}_{2}\left(\bar{\eta}_{2}-a\right)+a, b\left(1-\bar{\xi}_{2}\right)\right] ; \quad|J|_{2}=\bar{\xi}_{2} b \text { for } \Delta_{2},} \\
{[\xi, \eta]_{3}=\left[\bar{\xi}_{3}\left(1-a-\bar{\eta}_{3}\right)+a, \bar{\xi}_{3}\left(\bar{\eta}_{3}-b\right)+b\right] ; \quad|J|_{3}=\bar{\xi}_{3}(1-a-b) \text { for } \Delta_{3},}
\end{gathered}
$$

as well as for a quadrilateral element (subtriangles referred to as $\Delta_{l}$ ),

$$
\begin{gathered}
{[\xi, \eta]_{1}=\left[\bar{\xi}_{1}\left(\bar{\eta}_{1}-a\right)+a, b\left(1-\bar{\xi}_{1}\right)\right] ; \quad|J|_{1}=\bar{\xi}_{1} a \text { for } \Delta_{1},} \\
{[\xi, \eta]_{2}=\left[\bar{\xi}_{2}(1-a)+a, \bar{\xi}_{2}\left(\bar{\eta}_{2}-b\right)+b\right] ; \quad|J|_{2}=\bar{\xi}_{2}(1-a) \text { for } \Delta_{2},} \\
{[\xi, \eta]_{3}=\left[\bar{\xi}_{3}\left(1-a-\bar{\eta}_{3}\right)+a, \bar{\xi}_{3}(1-b)+b\right] ; \quad|J|_{3}=\bar{\xi}_{3}(1-b) \text { for } \Delta_{3},} \\
{[\xi, \eta]_{4}=\left[a\left(1-\bar{\xi}_{4}\right), \bar{\xi}_{4}\left(1-b-\bar{\eta}_{4}\right)+b\right] ; \quad|J|_{4}=\bar{\xi}_{4} a \text { for } \Delta_{4} .}
\end{gathered}
$$

The ratio $|J|_{l} / r\left(\bar{\xi}_{l}, \bar{\eta}_{l}\right)$ is always finite, which justifies the classically proposed coordinate transformation, but with the added advantage of enabling the analytical 
evaluation of the problem's key integrals [5]. As implemented, transformations end up carried out directly from Cartesian to subtriangle coordinates.

These developments are - to the authors' best knowledge - a novel generalization of a transformation classically proposed in the literature on finite and boundary element methods that uses a square-to-triangle degeneration in order to deal with a singularity [6]. The singularity of concern for the transformation is located at a point $\mathrm{P}(\xi=a, \eta=b)$, as in Fig. 1. For an analytical function $f(\xi, \eta)$ and the T3 triangle, integration is carried out over transformed spaces corresponding to the indicated subtriangles, $\widehat{\mathrm{P} 12}, \widehat{\mathrm{P} 23}$ and $\widehat{\mathrm{P} 31}$,

$$
\int_{0}^{1} \int_{0}^{1-\eta} f(\xi, \eta) \mathrm{d} \xi \mathrm{d} \eta=\sum_{l=1}^{3} \int_{0}^{1} \int_{0}^{1} f\left(\xi\left(\bar{\xi}_{l}, \bar{\eta}_{l}\right), \eta\left(\bar{\xi}_{l}, \bar{\eta}_{l}\right)\right)\left|J_{l}\left(\bar{\xi}_{l}, \bar{\eta}_{l}\right)\right| \mathrm{d} \bar{\xi}_{l} \mathrm{~d} \bar{\eta}_{l},
$$

considered as degenerated square subspaces $\left(\bar{\xi}_{l} \in[0,1], \bar{\eta}_{l} \in[0,1]\right), l=1,2,3$, where one of the nodal points of the unit square collapses in order to coincide with the point of singularity $\mathrm{P}$. The auxiliary coordinate systems $\left(\bar{\xi}_{l}, \bar{\eta}_{l}\right)$ have origin at node $\mathrm{P}$ and are oriented as indicated in Fig. 1. Depending on the depicted values of $a$ and $b$ one or two of these triangles may be void or correspond to negative areas, so that both cases of singularity and quasi-singularity are dealt with in the same algorithm. In the presently proposed general approach, the singularity may be located even outside the $(\xi, \eta)$ plane, as shown in Fig. 1(c) for a quadrilateral element represented in the Cartesian space and a singularity (source) point 0 . The distance from the source 0 to a field point $\mathrm{F}$ on the element is the hypotenuse $\overline{\mathrm{OF}}$ of the right triangle whose legs are the distances $\overline{\mathrm{OP}}$ from the source point to the plane and $\overline{\mathrm{PF}}$.

\subsection{Development for the case of a real quasi-singularity}

Although eqn (8) can be used in a straightforward way for improper integrals, the integral cannot be directly obtained for $0 \equiv \mathrm{P}$ when embedding a real singularity or hypersingularity. When $0 \equiv \mathrm{P}$ is outside the triangle segment, as for results at internal points that are close to the boundary and on the $(\xi, \eta)$ plane, one should better exclude the integration areas that are outside the actual integration interval, with eqn (8) evaluated according to one of the following particular cases (function arguments omitted):

$$
\begin{aligned}
& \int_{0}^{1} \int_{0}^{1-\eta} f \mathrm{~d} \xi \mathrm{d} \eta= \\
& \left\{\begin{array}{l}
|\tilde{J}|_{1} \int_{0}^{1}\left(\int_{\frac{b}{b+\bar{\eta}_{1}-1}}^{1}\left(f \bar{\xi}_{1}\right) \mathrm{d} \bar{\xi}_{1}\right) \mathrm{d} \bar{\eta}_{1}+|\tilde{J}|_{3} \int_{0}^{1}\left(\int_{\frac{b}{b-\bar{\eta}_{3}}}^{1}\left(f \bar{\xi}_{3}\right) \mathrm{d} \bar{\xi}_{3}\right) \mathrm{d} \bar{\eta}_{3} \quad \text { for } \quad b<0 \\
|\tilde{J}|_{2} \int_{0}^{1}\left(\int_{\frac{a}{a-\bar{\eta}_{2}}}^{1}\left(f \bar{\xi}_{2}\right) \mathrm{d} \bar{\xi}_{2}\right) \mathrm{d} \bar{\eta}_{2}+|\tilde{J}|_{3} \int_{0}^{1}\left(\int_{\frac{a}{a+\bar{\eta}_{2}-1}}^{1}\left(f \bar{\xi}_{3}\right) \mathrm{d} \bar{\xi}_{3}\right) \mathrm{d} \bar{\eta}_{3} \quad \text { for } \quad a<0 \\
|\tilde{J}|_{1} \int_{0}^{1}\left(\int_{\frac{a+b-1}{a+b+\bar{\eta}_{1}-1}}^{1}\left(f \bar{\xi}_{1}\right) \mathrm{d} \bar{\xi}_{1}\right) \mathrm{d} \bar{\eta}_{1}+|\tilde{J}|_{2} \int_{0}^{1}\left(\int_{\frac{a+b-1}{a+b-\bar{\eta}_{2}}}^{1}\left(f \bar{\xi}_{2}\right) \mathrm{d} \bar{\xi}_{2}\right) \mathrm{d} \bar{\eta}_{2} \text { for } \quad a, b \geq 0 .
\end{array}\right.
\end{aligned}
$$


Up to three cases are given in the first and third rows of expressions on the right-hand side: $a<0$ or $a=0$ or $a>0$ for $b<0$ as well as $(a=0, b>0)$ or $a, b>0$ or $(a>0, b=0)$ for $a<0$. One or two cases are comprised in the second row: $b=0$ or $b>0$ for $a<0$. Depending on $|\tilde{J}|_{l}$, as given in eqns (1)-(7), some integrals may be void or negative. The integration limit $\bar{\xi}_{l} \rightarrow 0$ is never reached.

Eqns (8) - for improper integrals and weak quasi-singularities - and (9) are compact sets of expressions directly needed in code implementations for potential and elasticity problems, provided that the integrals with integrands $\left(f \bar{\xi}_{l}\right)$ can be obtained analytically, which has been shown to be the case [5].

\section{GENERAL FORMULATION OF THE 3D PROBLEM}

For a linear triangle or a flat quadrilateral element, the evaluation of the coordinates $(a, b) \equiv\left(\xi_{\mathrm{P}}, \eta_{\mathrm{P}}\right)$ of Fig. 1 is a simple geometry matter. After a tedious development that uses the triangle to subtriangle transformations given in eqns (1)-(7), the distance square $\Delta r^{2} \equiv \overline{\mathrm{OF}}^{2}=\overline{\mathrm{OP}}^{2}+\overline{\mathrm{PF}}^{2}$ of Fig. 1 (see [5] for triangles and more generally) becomes expressed for each subtriangle as

$$
\Delta r_{l}^{2}=\Delta r_{0}^{2}+c_{l}^{2} \bar{\xi}_{l}^{2} \equiv \Delta r_{0}^{2}+\left(\tilde{a}_{l} \bar{\eta}_{l}^{2}+\tilde{b}_{l} \bar{\eta}_{l}+\tilde{c}_{l}-\Delta r_{0}^{2}\right) \bar{\xi}_{l}^{2}
$$

where $\Delta r_{0}^{2} \equiv \overline{0 \mathrm{P}}^{2}$. One checks that, for a triangle whose area is not void, $\tilde{a}_{l}>0$. Moreover, particular cases are $c_{l}^{2}=\tilde{a}_{l} \bar{\eta}_{l}^{2}$, when the singularity is located at node 2,3 or 1 for $l=1,2$ or 3 , respectively, as for the triangle element. Also, $\tilde{c}_{l} \geq 0$. Moreover, for a subtriangle $l=1,2,3$ and using a cyclic notation for $L(l):=3,1,2,3,1$, one obtains according to Fig. 1(a):

$$
\begin{aligned}
& \tilde{a}_{l}+\tilde{b}_{l}+\tilde{c}_{l}=\overline{0(L(l)-1)}^{2} \geq 0 \\
& \tilde{a}_{l}+\tilde{b}_{l}+\tilde{c}_{l}-\Delta r_{0}^{2}=\overline{\mathrm{P}(L(l)-1)}^{2} \geq 0 \text { for the triangle element } \\
& 4 \tilde{a}_{l}\left(\tilde{c}_{l}-\Delta r_{0}^{2}\right)-\tilde{b}_{l}^{2} \geq 0 .
\end{aligned}
$$

In the case of a singularity or real quasi-singularity, $\Delta r_{0}^{2} \equiv \overline{\mathrm{OP}}^{2}=0$ and the distance tends to zero with $\bar{\xi}_{l} \rightarrow 0$ independently from the respective coordinates $\bar{\eta}_{l}$. All particularities of subtriangles with void or negative areas (void or negative integrals) are taken care of by the Jacobian transformations given in eqns (1)-(7) as well as by developments according to eqns (8) and (9).

Eqn (10) - for flat triangle and quadrilateral elements (with straight edges, of course) is part of a general outline for high order, distorted elements, with radial distances given as

$$
\begin{aligned}
& \text { T3: } \Delta r_{l}^{2}=\Delta r_{0}^{2}+\bar{\xi}_{l}^{2} \sum_{j=0}^{2} c_{0 j} \bar{\eta}_{l}^{j} ; \quad \text { Q4: } \Delta r_{l}^{2}=\Delta r_{0}^{2}+\bar{\xi}_{l}^{2} \sum_{i=0}^{2} \bar{\xi}_{l}^{i} \sum_{j=0}^{2} c_{i j} \bar{\eta}_{l}^{j} \\
& \text { T6: } \Delta r_{l}^{2}=\Delta r_{0}^{2}+\bar{\xi}_{l}^{2} \sum_{i=0}^{2} \bar{\xi}_{l}^{i} \sum_{j=0}^{2+i} c_{i j} \bar{\eta}_{l}^{j} ; \quad \text { Q8: } \Delta r_{l}^{2}=\Delta r_{0}^{2}+\bar{\xi}_{l}^{2} \sum_{i=0}^{4} \bar{\xi}_{l}^{i} \sum_{j=0}^{\min (2+i, 4)} c_{i j} \bar{\eta}_{l}^{j} \text {, }
\end{aligned}
$$


where $c_{i j}$ are coefficients to be evaluated and adequately interpreted for their geometric representation, as done in eqn (11). It is still open whether such general problems may be dealt with in the present, analytical framework.

\section{EVALUATIONS FOR POTENTIAL PROBLEMS}

Owing to space restrictions, the explicit expressions for the single- and double-layer potential matrices $\mathbf{G}$ and $\mathbf{H}$ for 3D potential and elasticity are not given [1], [5]. The developments for arriving at such expressions are actually relevant to show the kind of integrals we must deal with as well as how important it is to take the properties outlined in Section 3 into account [5]. The results are briefly summarized in the following. All integrals could be evaluated analytically using the mathematical software Maple [7] and some ingenuity.

\subsection{Single-layer potential matrix $\mathbf{G}$}

For the single-layer potential matrix G [1], [5], the integrals

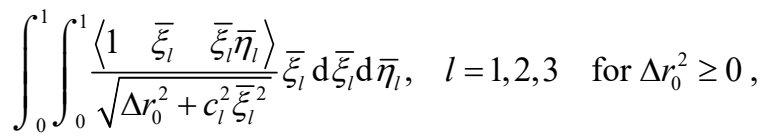

are required, where the array $\left\langle\begin{array}{lll}1 & \bar{\xi}_{l} & \bar{\xi}_{l} \bar{\eta}_{l}\end{array}\right\rangle$ contains the three terms of a bivariate polynomial that are relevant to the present developments. The integrals for $\Delta r_{0}^{2}=0$ (when $\bar{\xi}_{l}$ cancels out in numerator and denominator) and for $\Delta r_{0}^{2}>0$ are actually dealt with separately in the computational implementation.

\subsection{Double-layer potential matrix $\mathbf{H}$}

For matrix $\mathbf{H}$ and $\Delta r_{0}^{2}>0$, the analytical expressions of the three terms

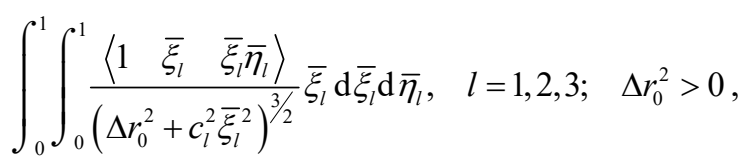

are required. For $\Delta r_{0}^{2}=0$ (potential problems), these integrals are void. For a diagonal term of $\mathbf{H}$, the finite part of the integrals are also void (for potential problems, but not for elasticity), but discontinuous terms to locally go around the singularity must be evaluated. This in principle involves a complicated geometry description of all elements that share a source point but could actually be dealt with in the frame of a simple algorithm [5].

\subsection{Results at internal points}

The evaluation of potential results at internal points requires the same types of integrals above. For the quasi-singularity case of $\Delta r_{0}^{2}>0$, the evaluation of gradient results deals with the analytical expressions of 12 integrals, schematically represented for $p=3$ and $p=5$ : 


$$
\int_{0}^{1} \int_{0}^{1}\left[\begin{array}{ccc}
1 & \bar{\xi}_{l} & \bar{\xi}_{l} \bar{\eta}_{l} \\
\cdot & \bar{\xi}_{l}^{2} & \bar{\xi}_{l}^{2} \bar{\eta}_{l} \\
\cdot & \cdot & \bar{\xi}_{l}^{2} \bar{\eta}_{l}^{2}
\end{array}\right] \frac{\bar{\xi}_{l} \mathrm{~d} \bar{\xi}_{l}}{\left(\Delta r_{0}^{2}+c_{l}^{2} \bar{\xi}_{l}^{2}\right)^{p / 2}} \mathrm{~d} \bar{\eta}_{l}, p=3 \text { or } 5, l=1,2,3 ; \Delta r_{0}^{2}>0 .
$$

For a real quasi-singularity, that is, $\Delta r_{0}^{2}=0$ for the source point outside the boundary segment but on its plane, one evaluates the five integrals

$$
\iint\left[\begin{array}{ccc}
1 & \bar{\eta}_{l} & \bar{\eta}_{l}^{2} \\
\cdot & 1 / \bar{\xi}_{l} & \bar{\eta}_{l} / \bar{\xi}_{l}
\end{array}\right] \frac{\mathrm{d} \bar{\xi}_{l}}{c_{l}^{3}} \mathrm{~d} \bar{\eta}_{l}, \quad l=1,2,3 \text { for } \Delta r_{0}^{2}=0,
$$

in the interval $[0,1]$, in principle, but making the adjustments indicated in eqn (9) for $\bar{\xi}_{l}$ in the denominator. For elasticity problems and triangle and quadrilateral flat elements, there are a few more polynomial terms in the numerators of eqns (13)-(16) and the denominators are affected by powers one order higher. However, all integrals may be evaluated analytically, as well [5].

\section{A QUEST FOR SIMPLIFICATION OF THE TRIANGLE ELEMENT T3}

Fig. 2(a) shows the element T3 for potential or displacement nodal values referred to the vertices, with degrees of freedom common to all elements that share a vertex (topological issues concerning fracture mechanics, for instance, not considered). Linear potential or displacement fields along the element are obtained by interpolation - exactly as in the isoparametric formulation of the displacement finite element method. On the other hand, the representation of normal gradients or traction forces, as for the evaluation of the singlelayer potential matrix $\mathbf{G}$, requires a framework that has no parallel in the (traditional) finite element formulation. Normal gradients and traction forces are surface attributes and cannot be attached to nodes. If we geometrically refer such attributes to an element's boundary point, this must be interpreted as a limiting case, as illustrated in Fig. 2(a). In such a case, normal gradients or traction forces are also linearly interpolated along the element in terms of the given surface attributes. A numerical model implemented with such an element must exactly (within machine precision) represent constant and linear potential or displacement fields. Since for a flat surface the corresponding normal gradients (and traction forces) have respectively zero and constant values, one might ask whether the use of a single parameter for such quantities - geometrically referred to the element's middle point, as in Fig. 2(b) would lead to reasonable numerical results. This will be illustratively assessed in the numerical simulation of Section 6. We also must keep in mind the total number of degrees of freedom involved in such numerical simulations.

Euler's theorem for a simply connected polyhedron is $V+F-E=2$, where $V$ is the number of vertices, $F$ the number of faces and $E$ the number of edges. It may also be shown that, in the special case of the simply connected polyhedron made up of triangular faces, $3 F=2 E$. Then, we obtain for the $\mathrm{T} 3$ element and a simply connected domain that $F=2 V-4$. If there are cavities in the domain, we must apply this formula to each separate cavity. On the other hand, if the domain is generally not convex, such as in a toroid, the relations above do not apply. Nevertheless, one may affirm that, when refining a 3D boundary with $\mathrm{T} 3$ triangle elements, the number of faces $F$, that is, elements, tends to be equal to twice the number of vertices $V$, that is, nodes: $F \rightarrow 2 V$ as $V \rightarrow \infty$. A similar reasoning leads to the conclusion that, for a boundary discretization with Q4 quadrilateral 


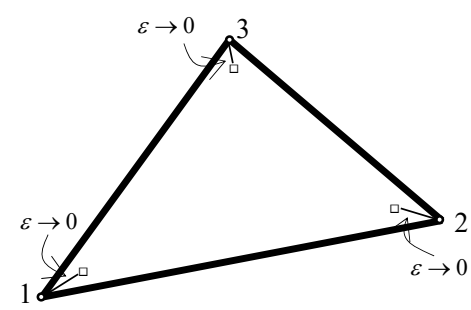

(a)

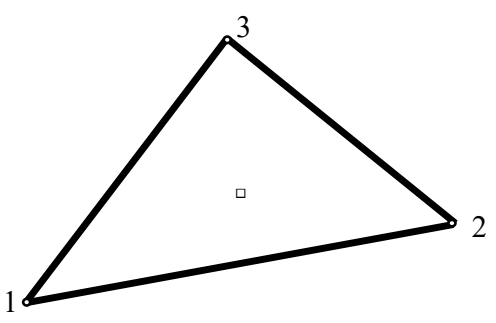

(b)

Figure 2: Element T3 with three nodal points for potentials (at the vertices) and surface attributes for normal gradients. (a) Three geometrically attached to points that tend to the vertices; and (b) One for a surface point referred to the triangle barycenter.

elements, $F \rightarrow V$ as $V \rightarrow \infty$. Then, in a numerical simulation of a potential 3D problem using T3 elements with three nodal parameters for potentials and three attributes for the normal gradients, the double-layer potential matrix $\mathbf{H}$ is always square and of order $V$, whereas the single-layer potential matrix $\mathbf{G}$ has $V$ rows and $3 F(\sim 6 V)$ columns, in general - both matrices of rank $V-1$ for a finite domain, if the problem is consistently formulated [2], [3]. This is the case of boundary condition discontinuities of the discretized surfaces that have a vertex in common. Observe that this assertion is more general and conceptually more appropriate than just saying that we are dealing with locally non-smooth surfaces. If one is sure that there are no boundary discontinuities of the normal gradients on the surface around a vertex, one may consider - for computational economy - one normal gradient degree of freedom at the considered vertex. In the case of only one normal gradient attribute geometrically referred to the middle of the T3 element, the number of columns of $\mathbf{G}$ is just $F$ and its rank is the same as before. (The dilemma continuous versus discontinuous elements is just a matter of misconception [2]).

\section{A SIMPLE NUMERICAL ILLUSTRATION}

Fig. 3 represents a very irregular $3 \mathrm{D}$ domain with a cavity (drawing rotated with angles $\vartheta=105^{\circ}, \varphi=80^{\circ}$ ), whose boundary is discretized with 16 linear triangle elements and 12 nodes. This is a development of the numerical example presented in [5]. The Cartesian coordinates of these nodes are

$$
\begin{aligned}
& \text { Coord }=\left[\begin{array}{cccccccc}
1 & 2 & 3 & 4 & 5 & 6 & 7 & 8 \\
\hdashline 1 & 1 & 0.2 & -1 & -2 & -1 & 0.5 & -0.25 \\
-0.5 & 0.4 & 0 & 0.5 & 0 & -0.5 & -1 & \frac{-0.55}{3} \\
0.5 & -0.5 & 1 & 1 & -0.2 & 0.5 & -1 & 0.05
\end{array}\right. \\
& \left.\begin{array}{cccc:cc:cccc}
9 & 10 & 11 & 12 & \overline{1} & \overline{2} & \overline{3} & \overline{4} & \overline{5} & \overline{6} \\
\hdashline-0.3 & -0.5 & -0.4 & 0 & -0.035 & 0.285 & -0.075 & 0.16 & 1.04 & -0.3 \\
-0.2 & -0.1 & -0.3 & 0 & \frac{-0.275}{3} & -0.5 & \frac{-0.605}{3} & -0.02 & 0.42 & -0.15 \\
0.3 & 0.4 & 0.5 & 0.6 & 0.475 & -0.05 & 1.055 & 1.075 & -0.575 & 0.45
\end{array}\right],
\end{aligned}
$$


using the first row for the node numbering, in an array that also contains the coordinates of internal points $\overline{1}, \overline{2}$ and external points $\overline{3}, \overline{4}, \overline{5}, \overline{6}$ at which potential and gradient results are to be evaluated. The nodal incidence is

$$
\text { Inc }=\left[\begin{array}{cccccccccccc:ccccc}
\frac{1}{2} & 2 & 3 & 4 & 5 & 6 & 7 & 8 & 9 & 10 & 11 & 12 & 13 & 14 & 15 & 16 \\
\hline 1 & 3 & 5 & 5 & 3 & 1 & 8 & 4 & 4 & 8 & 7 & 7 & 9 & 10 & 9 & 9 \\
2 & 2 & 3 & 6 & 6 & 3 & 2 & 2 & 8 & 6 & 6 & 8 & 10 & 12 & 11 & 12 \\
3 & 4 & 4 & 3 & 7 & 7 & 1 & 8 & 5 & 5 & 8 & 1 & 11 & 11 & 12 & 10
\end{array}\right] .
$$

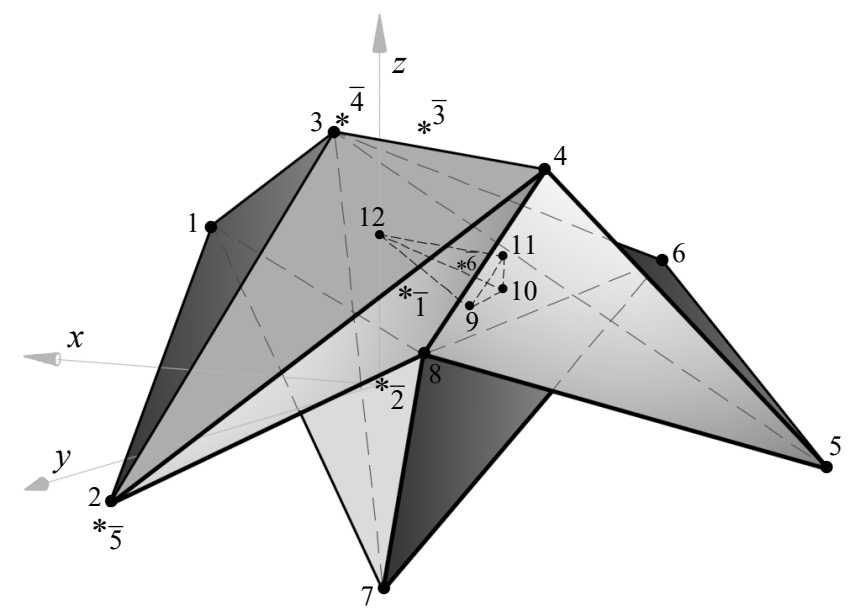

Figure 3: Very irregular domain with internal points $\overline{1}, \overline{2}$ and external points $\overline{3}, \overline{4}, \overline{5}, \overline{6}$ at which potential and gradient results are to be evaluated.

It is worth observing that elements 6 (nodes [ [ $\left.\begin{array}{lll}1 & 3 & 7\end{array}\right]$ ) and 12 (nodes $\left[\begin{array}{lll}7 & 8 & 1\end{array}\right]$ ) are almost coplanar. Nodes 9-12 correspond to a cavity built up with elements $13-16$. The external point $\overline{6}$ is precisely in the middle of the cavity. All nodes and internal and external points are very close to the boundary segments. For such a small example (no far nodes, for which a numerical quadrature would be preferable in order to save computational costs), the diagonal terms of $\mathbf{H}$ are analytically evaluated by just imposing orthogonality to a constant potential field. Consistency check of the boundary element equation $\mathbf{G t}=\mathbf{H d}$ for 3 linearly varying potential fields holds for 19 digits in an implementation using Maple software [7] with 20 digits of precision. The internal point $\overline{1}$ and the external points $\overline{3}, \overline{6}$ present complex quasi-singularities with respect to all 16 elements, with gradient results evaluated according to eqn (15). The internal point $\overline{2}$ is on the plane of element 6 and corresponding gradients must be evaluated for all three subtriangles as given in the second row on the right-hand side of eqn (9), for $a=-0.1, b=0.55$, with negative area for subtriangle 1 . The external points $\overline{4}$ and $\overline{5}$ are in the intersection of the planes given by elements 1 and 2 . Gradient results at point $\overline{4}$ require evaluations for element 1 ( $a=0, b=-0.05$ ) over subtriangles 2 (negative area) and 3 , and for element 2 
( $a=1.05, b=-0.05$ ) over subtriangles 1 and 2 (the latter with negative area), both cases according to the first row of eqn (9). Gradient results at point $\overline{5}$ require evaluations for element 1 ( $a=0, b=1.05$ ) over subtriangles 2 and 3 (negative area) according to the third row of eqn (9), and for element $2(a=-0.05, b=1.05)$ over subtriangles 1 (negative area) and 2 according to the second row of eqn (9). Then, all possible cases of quasi-singularity and topology issues are numerically assessed. Accuracy for potential results at all six points is checked for about 18 digits. For gradient results round-off errors unavoidably occur and accuracy is checked for at least 16 digits in the case of points $\overline{1}, \overline{2}, \overline{6}$ and at least 13,12 and 10 digits in the case of points $\overline{3}, \overline{4}$ and $\overline{5}$, respectively. (Results at external points $\overline{3}, \overline{4}, \overline{5}, \overline{6}$ obviously compare with zero.)

The domain of Fig. 3 is submitted to a total of 36 independent potential fields (solutions of the Laplace equation) corresponding to 1 constant, 3 linear, 5 quadratic, 7 cubic, 9 quartic and 11 quintic polynomials. The respective results measured on the boundary as nodal potentials and normal gradient attributes are applied to first check the consistency of the BEM equation $\mathbf{G t}=\mathbf{H d}$. This equation turns out to hold exactly (within machine precision) for the simplest four solutions. Fig. 4(a) shows the Euclidean norms of relative errors $|\mathbf{G t}=\mathbf{H d}|$ of this equation for the remaining 32 test solutions, vertical dash lines separating the polynomial orders. Four different kinds of results are grouped. The errors are in general very large, as the applied fields correspond to high degree polynomials and there are too many boundary irregularities for such a coarse mesh. The solid (black) and long dash (red) lines correspond respectively to the elements in Fig. 2(a) (3 parameters) and 2(b) (1 parameter) for the single-layer potential matrix $\mathbf{G}_{a d m}$ evaluated in the admissible way preconized by Dumont [2]. The dash-dot (black) and dash (red) lines correspond to the same results above, but with $\mathbf{G}$ evaluated as traditionally (and conceptually wrongly). For the 5 quadratic potential fields the measured normal gradients are in balance and there is no difference between admissible and inadmissible evaluations. As communicated in [3], there is no means of telling whether admissible evaluations should lead to more accurate solutions - this is observed in the graphic, in general, but exceptions are also shown. In this example, the matrix $\mathbf{H}$ is of order 12 and rank 11. The most relevant conclusions concern the matrix $\mathbf{G}$, which has 12 rows, but 48 columns for the element with 3 parameters, and only 16 rows for the element with just 1 parameter for normal gradients - for no numerically sensible accuracy loss, according to the graphics. Since there are no approximations in the integral evaluations, the differences in results are solely attributable to the different simulation schemes. The comparatively good results for the discretization with one element middle node for normal gradients is not a surprise, as the middle point is the optimal location for a quadrature scheme.

Out of the many numerical assessment possibilities for evaluations at internal (and external) points, Fig 4(b) and 4(c) refer to the internal point $\overline{1}$ of Fig. 3, with the same type of simulations described in Fig. 4(a) - together with the analytical values for the sake of comparison. The conclusions arrived at for the first graphic are here corroborated.

\section{CONCLUDING REMARKS}

The developments of this paper apply to steady-state potential and elastostatics problems implemented for T3 and flat Q4 elements. Owing to space restrictions only the T3 implementation for potential problems is outlined and numerically assessed with some 


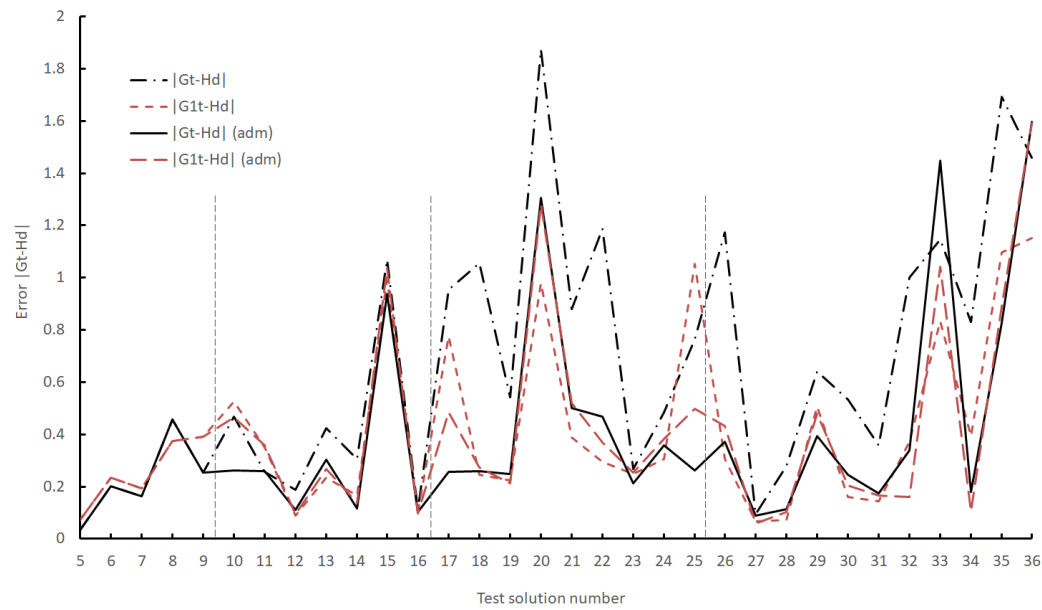

(a)

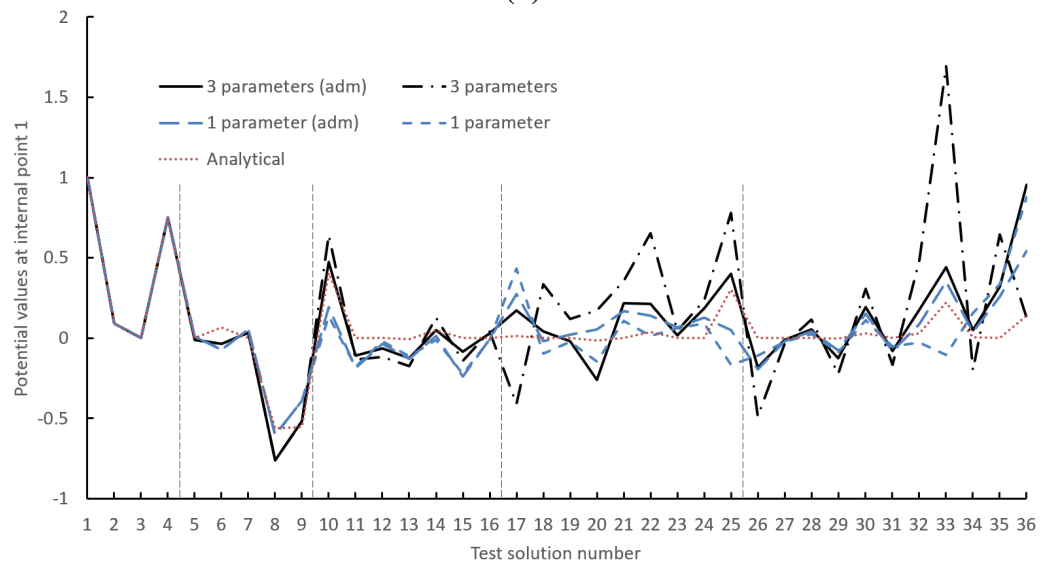

(b)

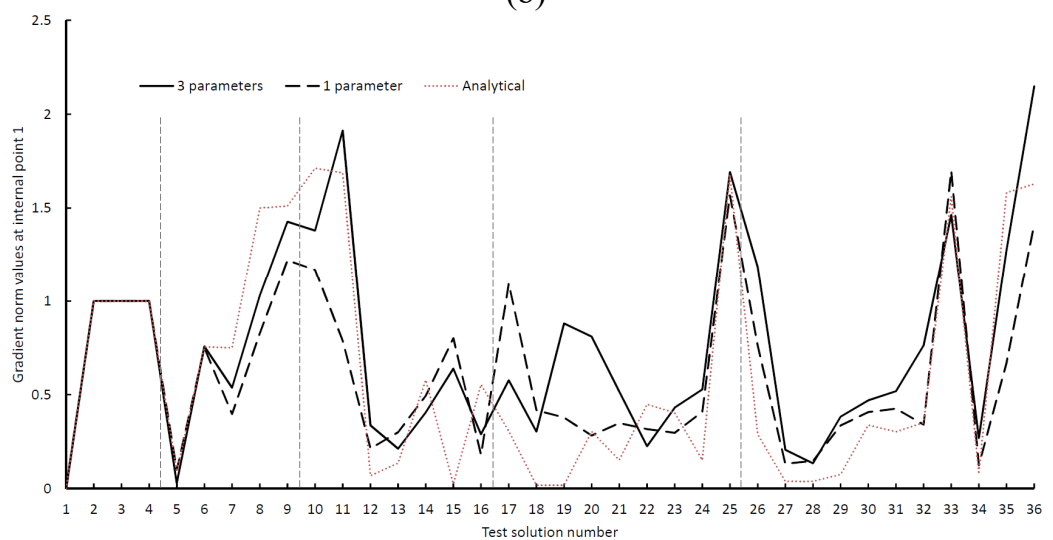

(c)

Figure 4: Some numerical results for the example of Fig. 3. (a) Consistency check of the equations; (b) Potential; and (c) Euclidean norm of gradient results at point $\overline{1}$. 
detail. All integrations are carried out analytically. The arrays with such analytical results will be published opportunely, but may be made available on request. Since there are no unsolved numerical integration issues, no matter how close a source point is to an element surface and how particularly located, it is for the first time possible to numerically assess that the use of just one middle node parameter for the boundary representation of normal gradients with the T3 element is of definitive computational advantage. It is also for the first time numerically illustrated that the concept of an admissible single-layer potential matrix, as preconized by the first author already in 1998, leads to better and more consistent results also for 3D problems than as outlined in the classical literature on boundary element methods.

\section{ACKNOWLEDGEMENTS}

This work was supported by the Brazilian agencies CAPES, CNPq and FAPERJ.

\section{REFERENCES}

[1] Brebbia, C.A., Telles, J.C.F. \& Wrobel, L.C., Boundary Element Techniques, Springer-Verlag: Berlin and New York, 1984.

[2] Dumont N.A., The collocation boundary element method revisited: perfect code for $2 \mathrm{D}$ problems. International Journal of Computational Methods and Experimental Measurements, 6(6), pp. 965-975, 2018.

[3] Dumont, N.A., An assessment of the spectral properties of the matrix G used in the boundary element methods. Computational Mechanics, 22(1), pp. 32-41, 1998.

[4] Dumont, N.A., On the efficient numerical evaluation of integrals with complex singularity poles. Engineering Analysis with Boundary Elements, 13, pp. 155-168, 1994.

[5] Kurz, T.G., Analytical 3D Boundary element implementation of flat triangle and quadrilateral elements for potential and elasticity problems, $\mathrm{PhD}$ thesis, in progress, in Portuguese, PUC-Rio, Brazil, 2019.

[6] Lachat, J.C. \& Watson, J.O., Effective numerical treatment of boundary integral equations: A formulation for three-dimensional elastostatics. International Journal for Numerical Methods in Engineering, 10(5), pp. 991-1005, 1976.

[7] Maplesoft, a division of Waterloo Maple Inc., Maple 15, Waterloo, Ontario. 